\title{
Immaginare e visualizzare la monarchia in Sicilia. La cultura materiale della corona normanna
}

\author{
Antonio Pio Di Cosmo \\ (ISACCL, Bucarest. Universidad Católica de Cuyo). \\ apiocosmo@outlook.it \\ Imagine and visualize the monarchy in Sicily. \\ The material culture of the Norman Crown
}

RECIBIDO: 2I-05-202I

EVALUADO Y ACEPTADO: 2I-09-202I

Territorio, Sociedad y Poder, No I6, 202I [PP. 23-4I] 
Riassunto: Il contributo analizza il ruolo giocato dai loci di status, come l'utilizzo della formula del «a Deo coronatus», la progettazione di un abbigliamento fastoso ed il ricorso al porfido per descrivere la monarchia siciliana. Questa ricerca vaglia le strategie di comunicazione orientate al timor reverentiae,

Авsтract: This contribution analyzes the role of status' loci and the use of the "a Deo coronatus" formula, the design of sumptuous clothing, and the use of porphyry, those are utilized to describe the Sicilian Monarchy. This inquiry scrutinizes communication's strategies of timor reverentiae, that che insufflano precise idee nei sottoposti. Questi espedienti rafforzano così la percezione di una monarchia derivante direttamente da Dio.

Parole chiave: re di Sicilia, Altavilla, «a Deo coronatus», abbigliamento, porfido.

infuse precise ideas into the subjects, and so those gimmicks reinforce the perception of a monarchy, which derives directly from God.

KEYwords: king of Sicily, Altavilla, "a Deo coronatus», clothing, porphyry. 


\section{INTRODUZIONE}

Ruggero (...). Andò dal re [Luigi VI di Francia], e gli propose di percorrere con lui tutto il suo castello, di vedere il suo tesoro e di prendere in abbondanza quanto gli fosse piaciuto, come se fosse stato suo (...). Il re si recò dunque con lui al suo tesoro (...). Ruggero prese una corona d'oro molto bella, andò dal re e gli disse: "Signore, vi piacerebbe mettere questa corona d'oro sulla mia testa, per sapere come mi starebbe?” (...) Il re (...) prese la corona in mano e gliela mise sulla testa. Quando Ruggero ebbe la corona sulla testa (...) lo ringraziò moltissimo e disse che non l'avrebbe potuto mai incoronare un uomo più eccelso di lui (...). E il re Ruggero lo accompagnò fino al mare e fece portare con lui gran parte dei suoi gioielli (Chronique d'Ernoul et de Bernard le Trésorier, I3-I4, trad. it. Delle Donne, 2006: 332).

Il passo della Cronaca di Ernoul e di Bernardo Tesoriere, che si apprezza nella recente traduzione di Fulvio Delle Donne, illustra un locus diffuso e condiviso per descrivere la vita della corte palermitana: l'estremo lusso e l'abbondanza di risorse che si declinano come cifra di stile. ${ }^{1}$ Nondimeno, offre un'interpretazione fantasiosa della nascita della monarchia normanna, che poco ha a che fare con gli eventi storicamente verificabili. Propone piuttosto al pubblico un'interpretazione degli accadimenti che circondano la promotio di Ruggero II. L'aneddoto dimostra come il contesto internazionale percepisca la creazione della nuova monarchia e la liquida quale forzatura, giacché attuata a mezzo di pressioni e con la complicità dell'antipapa Anacleto. Non meraviglia allora che compaia un locus che contrappone l'astuzia di Ruggero all'anima priva di malizia del re francese. Un inganno, se mai avvenuto, realizzatosi con probabilità intorno al IIzo e per di più perpetrato ai danni di un uomo fornito di una auctoritas tale da riempire di ulteriore significatività una prossemica di per sé altamente significante, ma altresì priva di ogni valore giuridico ${ }^{2}$. Il gesto forse costituisce un'eco dell'incoronazione effettuata da parte del principe di Capua, come riferito da Falcone di Benevento, che accantona il "tocco" di un rappresentante della Chiesa (Falcone di

\footnotetext{
${ }^{1}$ L'episodio viene pure citato in modo sommario in: Gesta Henrici II et Ricardi I, I, 6-II; Iohannis Brompton, Chronicon, col. IoII.

2 Il gesto suscita perplessità, il "tocco" di un pari evoca solo l'auctoritas di Ludovico ed il suo prestigio personale, ma dal punto di vista del diritto il gesto non ha alcuna validità de jure. È ben noto che per il diritto tardoantico e alto medievale nemmeno un re ha il potere di creare un suo pari. Tale facoltà è esclusiva competenza imperiale e poi papale. Cfr. Zug Tucci, 2002.
} 
Benevento, Chronicon Beneventanum, II3O, 4, I). Compare anche il tema della prodigalità di Ruggero, quale leitmotiv. Un dispendio a maggior ragione giustificato entro i riti di accoglienza, che per consuetudine sociale esigono tale devolumento di risorse private. Nonché ha ragioni politiche e vuole suscitare ammirazione ed un timor non modicus nell'esponente del gruppo dei pari. ${ }^{3}$ Eppure, l'aneddoto stempera l'accadimento in un clima favolistico, che rimanda con buona probabilità a quell'abbondanza profusa durante i riti di ascesa di Ruggero. Sicché si raffronta una riscrittura creativa e politicamente orientata della storia. Quel che conta, difatti, è suggestionare il pubblico, mentre l'immaginazione dell'entourage rougeriano evoca alla mente degli episodi connotati dall'ostentazione di un'eccezionale abbondanza (Duranti, 2019). Le strategie d'opulenza, orbene, costituiscono un leitmotiv condiviso per rappresentare l'esperienza monarchica di Ruggero II (Dawn, 2020). ${ }^{4}$ Una soluzione che deve rispondere al vero, tanto che viene presentificata pure dal poeta arabo Ab dar Rahman Muhammad ibn 'Umar. Costui descrive in modo suggestivo la vita di corte di Palermo e permette di visualizzarne la straordinaria prosperità: un principato che s'innalza/ sopra quello dei Cesari (...)./ Allāh l'ha colmato d'abbondanza... (Ab dar Rahman Muhammad ibn 'Umar, Yalla Harrek, vv. 8-9, II).

Orbene, la presente indagine procede da questi saporosi aneddoti che sviluppano dalle complesse soluzioni proposte dagli intellettuali assoldati da Ruggero II, i quali sono onerati del compito di immaginare una facies persuasiva e convincente per l'istituenda monarchia. Difatti, quei prodotti dell'immaginazione sono appositamente pensati per aiutare i sottoposti, ma anche il gruppo dei pari a visualizzare la formula prescelta per rappresentare il re locale. Pertanto, si

\footnotetext{
${ }^{3}$ Si nota che in Francesco Pipino compaiono i temi essenziali meglio sviluppati da Bernardo Tesoriere: l'astuzia e l'ostentazione quasi rituale delle ricchezze, connesse al gesto antropologicamente connotato del dono. Cfr. Francesco Pipino, Chronicon, coll. 587-752.

${ }^{4}$ Giovanni di Salisbury definisce l'orientamento dato da Ruggero II alla propria esperienza monarchica: adeo gloriatur ut palam dicat se nunc demumavi sui consecutum privilegium, qui in terra sua erat rex, legatus apostolicus, patriarcha, imperator et omnia quae volebat..., cfr. Ioannis Saresberiensis Epistolae 239. Vedia anche Carver y Molinari, 2020.
}

considerano le implicazioni di simili elaborazioni sulla cultura materiale connessa alla fenomenologia della regalità. Perseguendo tale fine esegetico si collazionano quelle informazioni indispensabili a visualizzare il monarca, che sono fornite dai documenti visuali prodotti dalla dinastia degli Altavilla, in particolare dalle opere monumentali, nonché dalla cultura materiale della regalità e, più segnatamente, delle singole insegne che caratterizzano l'epifania del re siciliano. Infine, si esaminano i segni più duraturi della potestà regia: $i$ sepolcri, i quali nel lungo periodo affermano i valori della monarchia.

Tali prodotti della cultura materiale sono difatti oberati nella dinamica del ménage quotidiano del Regno di Sicilia del compito di rappresentare il carisma detenuto dal re locale e di indurre gli avventori ad una maggiore consapevolezza dello stesso, fungendo da "garanzia materiale" della legittimità dell'Istituzione. Se ne valorizza così la capacità semiologica, volta a visualizzare i messaggi sottesi ai medesimi prodotti e, più in generale, quell'attitudine a spiegare in modo relativamente semplice le complesse elaborazioni dell'immaginazione degli intellettuali. I prodotti della cultura materiale vengono dunque adoperati quali strumenti di hexis nell'esegesi delle strategie di autorappresentazione della monarchia, tant'è che costituiscono degli incunaboli per l'interpretazione delle soluzioni immaginate per raffigurare il re ed impiegate per meglio visualizzare il di lui corpo e la persona (Vagnoni, 2019).

La regalità degli Altavilla si costruisce come sorta di puzzle che immagina una serie di lemmi utili ad ottimizzare l'efficacia della propria propaganda ed a rendere performante l'epifania del monarca. Un puzzle complesso di cui i politologi sono i produttori ed alla cui costruzione i sovrani sovrintendono. Un'operazione che pone in essere una collazione dei lemmi dell'idioma della regalità e tesaurizza esperienze di diversa ascendenza ed in particolare loci propri del potere della $\mathrm{Ba}$ sileia romano orientale (Houben, 1999). La formula descrittiva proposta dagli Altavilla costituisce un prodotto politico, che pone in essere un racconto concernente i valori della propria monarchia connotato da un chiaro valore polemico. L'incoronazione tradotta in 
forma "mistica" ed il presunto uso del loros, che realizza visivamente la christomimesis, rimandano ad un potere derivato direttamente da Dio. Il porfido poi traduce nella pietra lo stesso concetto di impero e si oppone alle pretese papali sul Regno di Sicilia. Si contrappone altresì avverso la Basileia a cui vengono sottratti i territori del Meridione d'Italia, nonché replica ai diritti vantati dall'Impero occidentale sull'area fino alla Calabria. La riproposizione delle morfologie romano orientali vuol manifestare la plenitudo potestatis di un monarca che pretende di essere in rapporto diretto col Cristo, da cui viene ogni potere, quale soluzione legittimante, poiché avvalla le aspirazioni autocratiche d'un officio che è però qualificabile dal punto di vista del diritto come derivato. Eppure, queste formule costituiscono meri trucchi affabulatori e di persuasione, ${ }^{5}$ giacché vanno incontro alle aspettative del pubblico e richiamano loci graditi e ben presenti alla memoria collettiva (Vagnoni, 2012).

\section{L'“A DEO CORONATUS»: UN ESPEDIENTE PER VISUALIZZARE LA REGALITÀ DIVINA DEGLI ALTAVILLA. LE SOLUZIONI DELLA CULTURA VISUALE E DELLA RETORICA}

Lo sforzo richiesto nell'immaginare una forma di monarchia autocratica e non derivata da alcuna autorità umana spiega il ricorso alla formula del «a Deo coronatus», già consueta nelle politiche di autorappresentazione della "teologia del potere" romano orientale, poiché connette il sovrano locale direttamente alla divinità cristiana e permette di visualizzare tale relazione. Questa viene proposta in modalità monumentali a Palermo da un esponente della cultura bizantina come l'ammiraglio Giorgio di Antiochia per essere così metabolizzata ed indi effettivamente visualizzata dal pubblico. Una

\footnotetext{
${ }^{5}$ Il contesto locale presenta un pubblico con l'occhio assuefatto a formule di rappresentazione romano orientale. E se la popolazione grecofona del Regno non sembra superare il $20 \%$ del totale, deve considerarsi un più ampio sostrato di cultura greca che spiega sia la buona ricettività, sia la propensione ad accettare una determinata formula, apprezzabile nei termini di "domanda sociale".
}

formula riproposta nonostante la progressiva desacralizzazione della figura regia nell'Europa del periodo di riferimento. Un processo di interiorizzazione della soluzione da parte della monarchia che procede dal II39, anno della costituzione del Regno, al II77, anno del riconoscimento da parte dell'Impero d'Occidente a mezzo del trattato di Venezia. Il locus risponde poi ad un'ulteriore esigenza, giacché appare capace di stabilire «gerarchie di supremazia» e di poter impedire, almeno a livello mentale, ogni possibilità di rivolta (Andenna, 2006: 37I-372). Sicché la conquista di una sacralità da parte del sovrano, resa percepibile agli occhi dei sottoposti dal documento visuale, deve essere apprezzata piuttosto come il prodotto di un «fenomeno storico, -e- storicamente elaborato», frutto di «operazioni intellettuali e culturali», che non può liquidarsi come mero "repertorio" altomedievale (Cantarella, 1996: 9-38). Il ricorso a questa soluzione aulica costituisce un atto politico altamente meditato e si oppone ai presupposti in diritto che fondano la propria esperienza monarchica: l'infeudazione papale del territorio e la concessione della promotio regia, nonché il rito d'ascesa realizzato a mezzo della consacrazione operata materialmente dai vescovi (Andenna, 2006). Eppure Ruggero non può prescindere da quel sistema di comunicazione che sfrutta l'azione dell'episcopato e propone una serie di segni, per il cui tramite si afferma fortemente la sacralità del sovrano locale. Ciò suscita consenso e lo pone in uno "spazio di copertura sociale" in cui opera la religione con i suoi valori. Fra queste forme simboliche rientra l'introduzione del rito dell'unzione, che rende il rappresentante adeguato al carisma della monarchia, ponendolo in effettivo rapporto con Dio.

Stanti tali presupposti, si comprende come la soluzione proposta da Giorgio di Antiochia, non sia liquidabile come un'improvvisazione, ma risponda alle problematiche di legittimazione vissute sul piano internazionale dal Regno. Tuttavia, tale intervento appare piuttosto come una forzatura, poiché recenti studi sembrerebbero dimostrare che il sovrano ha una "normale" considerazione di sé e dell'officio che riveste (Vagnoni, 20II). L'effige commissionata da Giorgio di Antiochia è eseguita a mosaico fra gli anni II43-II46 ed allocata 
nella chiesa di S. Maria, cosiddetta dell'Ammiraglio o Martorana, un edificio adibito originariamente a cappella di famiglia e mausoleo (Vagnoni, 20II; Kantorowicz, 1950; Kantorowicz, 1976; Kantorowicz, 1990; Kantorowicz, 2000; Reilly, 2020). La funzione privata dell'aula la destina così ad un pubblico elitario. Ivi si rappresenta Ruggero II in piedi, in atto di devozione ed in posizione speculare al Cristo, il quale sta ritto e gli impone la corona, mentre fluttua nell'oro. Nonostante la scena preveda entrambi i personaggi di tre quarti ed impegnati ad interagire, il loro sguardo si rivolge all'osservatore secondo una consuetudine tutta bizantina. In ossequio all'aspettativa sociale si adopera pure il criterio gerarchico delle proporzioni, sicché il Cristo sembra sovrastare Ruggero II. I personaggi sono identificati come di consuetudine dai tituli. Ruggero II è accompagnato dalla didascalia greca: РОГЕРІОС РН $\Xi$ (Ruggero re), invece il Cristo presenta l'abbreviazione: IC XC. Il re si mostra quale basileus e veste il divitision blu con clavi dorati e si orna di un loros a forma di Y, quale foggia residuale, che cita la moda della corte macedone. La posa del re che china il capo in segno di reverenza e offre preghiere ripropone un locus descrittivo adoperato per l'avorio rappresentante l'incoronazione mistica di Costantino VII. Il Cristo altresì veste il kithòn di porpora con clavi aurei ed il maphorion azzurro, mentre indossa stranamente dei pedilia purpurei. La divinità stringe nella mano sinistra il rotolo della legge ed il suo nimbo è crocesegnato. Giorgio vuole veicolare un messaggio compiacente e lapalissiano, allorché tenta di tabuizzare ogni riferimento al giuramento di vassallaggio offerto al papa, mentre propone al pubblico il legame diretto esistente fra Dio e il sovrano, cosa che rende residuale la mediazione della Chiesa.

Quel documento visuale "non" ufficiale e prodotto da un privato, ponendosi quasi a contrappunto della retorica ufficiale, permette di visualizzare una formula assimilabile e mutuata in un testo fondamentale della storiografia normanna: la Ystoria Serenissimi Rogerii Primi Regis Siciliae di Alessandro abate del monastero di Telese. Ivi si propone un'apologia politica e si dichiara nell'Alloquium ad regem Rogerium che è solo il volere divino a concedere sia il trono, sia la vittoria: memor sis Domini Salvatoris tiri regis aeterni, eique placere studeas cuius benefici munere te triumphasse, regnumque obtinuisse non dubitamus (...). Et pro Domino Salvatore Nostro (...) consecutus es regnum (Alexander Telesinus, Ystoria Serenissimi Rogerii Primi Regis Siciliae, 5, II; 2; D’Angelo, 2003). La riapparizione del locus della "Teologia della Vittoria» non solo offre una base di legittimità, ma funge da potente contrappeso, poiché dequalifica il re a mero esecutore della volontà divina, tanto da restringerne l'arbitrio (Gagé, I933: I-43). Questo perché ogni volta che si prende coscienza della "superpersonalità" afferita al sovrano, vi è la necessità di opporvi dei calmieri. Paradossalmente questi hanno a rafforzare la percezione della concezione che il sovrano ha di sé nel momento stesso in cui la limitano o addirittura la negano. La nuova fase vitale spiegata dal locus in Sicilia dimostra la capacità di diffusione degli episodi del potere della Basileia e di penetrazione per osmosi da contatto. L'elaborazione è riproposta da Filagato da Cerami nell'omelia pronunciata nella festa dei SS. Pietro e Paolo: a lui mantenga lo scettro in pace e tranquillità lo stesso Cristo che glielo ha dato (Filagato da Cerami, Omelia LV). ${ }^{6}$ Lomelia per la Festa delle Palme poi ribadisce la derivazione celeste del potere regio: il quale ornò questi con la sapienza, con la forza e con il diadema regio (Filagato da Cerami, Omelia XXVII). Le omelie di Filagato, un'intellettuale educato nel pensiero romano orientale, rifunzionalizzano simili loci nel contesto locale. A maggior ragione allorché si utilizza l'epiteto di $\beta \alpha \sigma \imath \lambda \varepsilon v ́ \varsigma$ per identificare Ruggero, a suo tramite poi si dichiara l'assimilazione delle facoltà proprie dell'imperatore romano orientale e la sua conseguente sostituzione nella guida del Meridione italiano. La coscienza di una monarchia uranica viene riproposta in una collezione di leggi come l'Assise di Ariano e specie nel suo proemio, laddove si ripropone il locus di un sovrano legibus solutus. Ciò è dopotutto un corollario dell'investitura divina, poiché il re deve dare conto a Dio solo del suo operato. Una potestà assoluta che anche questa volta richiede opportuni calmieri, giacché

\footnotetext{
${ }^{6}$ La predica è pronunciata forse nel II40 in occasione dell'inaugurazione della cappella palatina del palazzo palermitano o nella festa del II $43 \mathrm{O}$, ancora, più tardi nel II53.
} 
la sua azione deve essere orientata dai principii della iustitia, aequitas e misericordia (Delogu, 1983).

La convinzione concernente la derivazione divina del potere regio emerge chiaramente dal Corpus diplomatico ruggeriano e si evince dalle formule retoriche che compaiono nell' intitulatio, nella subscriptio e specie nell'arenga, laddove si lascia spazio a considerazioni più esplicite, allo scopo di persuadere i destinatari dei diplomi di una realtà a cui non si ammette deroga (Brühl, 1987; Brühl, Giunta, Guillou, 1987; Vagnoni, 20I2: IO2-IO7; Di Cosmo, 20I8). Dal II36 compare nella datatio e nella rota dei diplomi latini la formula ufficiale: Dei gratia Sicilie, Apulie et Calabrie rex, formula che conosce un'espressione alternativa: divina favente clementia rex Sicilie, ducatus Apulie et principatus Capue. Nell' intitulatio dei diplomi greci invece si ritrova solitamente l'espressione: in Cristo Dio pio potente re, che ritorna altrettanto sovente nella subscriptio. Citazioni che evocano la monarchia divina appaiono ancora nelle arenghe e nel corpo del testo: In regni regimine, Domino disponente, promoti conspicimur ed ob amorem regis celestis, per quem subsistimus et regnamus, nonché nos igitur, cui Deus in regni Sicilie primo solio voluit presidere o, persino, qui nobis et honorem contulit et nomen nostrum laude regia decoravit. Tali formule sono presenti nei diplomi latini di Ruggero II nn. I6, 38,43 e 68. Si ricorda ancora la più sintetica formulazione: Nobis a Deo concessa o la prolissa: que nobis omnipotentis Dei misericordia habere concessit entrambe rinvenibili nei diplomi latini nn. 3I, 43 e 48 emessi da Ruggero II. Un'idea a cui si fa riferimento anche nei diplomi in lingua araba: i jarda (Johns, 2006: II9-I3I). Le formule retoriche, sebbene non risultano formalmente assimilabili a quelle dei diplomi greci e latini, utilizzano epiteti culturalmente connotati e riconducibili al tenore della monarchia divina, appellativi quali laqab e alqb. ' La propaganda propone alla componente araba formule speculari al locus del «a Deo coronatus»,

\footnotetext{
7 Tali diplomi non sono redatti dalla cancelleria regia ma da un apposito ufficio: il $d w n$. Un organo regio esterno, che è titolare di una facoltà di agire autonoma ed è affidatario di mansioni amministrative peculiari. Non a caso, $i$ testi che da qui fuoriescono si riferiscono al sovrano menzionandolo sempre in terza persona.
}

che vengono iscritte nei tarì emessi da Ruggero II, quali il potente per grazia di Allāh o, ancora, il bramoso di potere per grazia di Alläh. Formule che si affiancano a IC XC NIKA. Ruggero opta per la riproposizione di una sorta di relitto della teoria del potere entro un contesto politico che ha subito le conseguenze della Lotta alle investiture. Eppure, quella formula appare indispensabile e costituisce una "clausola di salvaguardia" della monarchia, giacché volta a persuadere i sottoposti che ricevono gli esemplari del suo corpo diplomatico con un'idea precisa, la quale non ammette alternativa.

Nonostante non ci siano note rappresentazioni di Guglielmo I che ripetono il locus del "a Deo coronatus», l'idea di una monarchia uranica traspare chiaramente dal suo Corpo diplomatico (Vagnoni, 20I4). Possiamo così rinvenire espressioni quali Regii diadematis nostri primitias in ipsa et ab ipsa suscepimus, riscontrabile nel diploma n. 27. Nel diploma n. 9 si può evincere un'articolata dichiarazione che evoca la costante presenza di Dio a guida dell'azione del re: inter actus nostros, et operum dispositionem, que Rex regum omnium, et dominantium dominator à primordiis nostri regiminis clementer direxit, et misericorditer custodivit, (...) quo propitiante tranquillum nostrum regnum in pace fovetur, et omnes eminus turbines propelluntur. Eppure a quest'idea di matrice platonica, si affiancano elaborazioni di tipo aristotelico, allorché si ricorda come il massimo potere debba essere concesso al migliore. Eugenio di Palermo, che scrive in lingua greca il carme XXI intitolato $D e$ regno, afferma: di razza tanto superiore e preminente (...) -sicché non potendo- trarre giù dall'alto i principii immateriali sovramondani, perché vengono a regnare necessariamente li eleggiamo fra noi e li poniamo a governare su di noi col nostro consenso (Eugenii Panormitani Versus iambici, Poema XXIV). ${ }^{8}$ Tale riflessione si potrebbe porre alla fine di un lungo processo di desacralizzazione della maestà, che si consuma nel dibattito teorico e nella lotta fra papato ed Impero d'Occidente. Eppure Eugenio non si limita a ricordare che l'istituzione mo-

\footnotetext{
${ }^{8}$ Eugenio è un intellettuale attivo presso la corte palermitana, dove riveste le qualifiche di Magister della reale Duana baronum dal II74 al II89 e, poi, di Admiratus dal ııo al Iı95. Infine è eletto Magister camerarius delle Puglie e della Terra di Lavoro dal II98 al I202.
} 
narchica ha un'origine tutta umana, ma vuole indicare i criteri da utilizzare per riconoscere le qualità di colui che è degno del titolo di re. Siamo dunque di fronte ad un tentativo tutto laico di "messa sotto copertura" dell'istituzione e del suo rappresentante, che si declina nel segno dell'eccellenza del detentore del potere.

Il locus del «a Deo coronatus» viene riproposto fra il II74 ed il II86 in un documento visuale ufficiale. Poiché commissionato direttamente da Guglielmo II (II53-II89) e collocato presso la cattedrale di Monreale. Il re è rappresentato mentre si approssima a Cristo che lo incorona. Eppure quest'immagine dall'alto valore comunicativo ed atta a rendere visualizzabile tutto il carisma soprannaturale proprio del sovrano, ha un impatto piuttosto ridotto, dacché è percettibile esclusivamente a coloro che transitano nella navata centrale. Tuttavia, appare ben visibile al clero officiante, il quale prima di tutti deve essere persuaso circa la natura divina del potere di Guglielmo II e della presenza di Cristo nelle sue azioni (Ordo A, 31; Elze, 1973; Cantarella, 1996). Si inscena un vero e proprio atto di investitura che recupera la prossemica della divinità in trono, a cui si ha facoltà di avvicinarsi con devozione (Delogu, 1983; Kantorowicz, 1960). L'immagine si presenta ben più complessa del precedente della Martorana, costituendone un'evoluzione che segna la sua nuova vita nel locale, giacché si sommano due angeli che piombano giù dal cielo e consegnano al sovrano le insegne minori del potere: il labaro, emblema tipico del basileus, ed una sfera con incisa una croce. Il Cristo è identificato dal nimbo crucigero e dai propri tituli greci, sta assiso poi su un trono a cassone corredato di suppedion e di pulvinares, presentandosi quale Dominus. Questi osserva lo spettatore cercando il dialogo, mentre veste l'azzurro e la porpora. Nella sinistra tiene l'Evangelo che afferma: EGO SVM LVX MV[ndi] (GV 8, I2). Guglielmo II invece è accompagnato dall'epigrafe: REX GVILIELMVS S[E] $\mathrm{C}[\mathrm{VN}] \mathrm{D}[\mathrm{VS}]$ e si rivolge al Cristo magniloquente e rappresentato secondo dimensioni gerarchiche (Vagnoni, 20I2). Il re veste il divitision blu decorato in oro ed al di sotto indossa uno skaramangion bianco, che evoca l'alba da lui introdotta fra i vestimenta regalia, nonché il loros del tipo ad Y, mentre calza i pedilia pur- purei. La scelta nell'abbigliamento sembra proporre una mediazione tra il locus consueto adoperato per tradurre la veste del basileus ed i concreti vestimenta regalia, senza suscitare spaesamento visivo nel pubblico. Si aggiunge una novità estranea alla tradizione, consistente nell'inserimento sul fondo d'oro di una didascalia, che proferisce: MANVS ENI[M] MEA AVXILIABITVR EI. La dicitura che evoca il Salmo 88, versetto 22, ed una profezia di Natan ha molte implicazioni e propone un nesso messianico ed escatologico fra la regalità di Guglielmo e quella di Davide, giacché questi può essere identificato con lui in quanto rex juvenes.

Una certezza, quella dell'origine divina del proprio potere, che torna anche nei diplomi emessi da Guglielmo II. Nel diploma B 203, destinato alla regina Giovanna, si considera come il diritto divino al regno segua il criterio del sangue e debba essere trasmesso alla prole. Nonostante tale dichiarazione di intenti, si nota lo scemarsi della presenza di tali soluzioni nel suo Corpus diplomatico. Una differente percezione della regalità che non osta però all'inserimento di formule altamente evocative. Il diploma n. 9I dichiara non solo che il regno viene acquisito col consenso divino, ma la stessa divinità sostiene l'azione regia mediante l'ispirazione dell'operato. Un'origine celeste del potere terreno che viene ancora esaltata nel diploma n. 204, allorché si afferma: in regum operibus constat esse precipuum. Simili concetti sono ribaditi nei diplomi B 20I e B 220. Lidea concernente una concreta monarchia uranica viene declinata nelle formule magnificatorie in arabo, laddove si ritrovano dizioni come il sacro, il bramoso di potere per grazia di Alläh o il soccorso dalla Sua onnipotenza e, persino, il vittorioso attraverso la Sua forza (Vagnoni, 20I2).

III. LE VESTI ADEGUATE A VISUALIZZARE IL

CARISMA DEL SOVRANO SICILIANO: LE ONDE MNEMONICHE DEL COSTUME ROMANO ORIENTALE E L'IMMAGINAZIONE DEGLI INTELLETUALI LOCALI

Per quel che riguarda l'esperienza degli Altavilla, si può dire che è davvero la veste a fare il re, quale segno di eccellenza in sé e per sé. Non meraviglia allora che gli 
intellettuali locali hanno ad immaginare delle strategie che si orientano verso l'opulenza delle epifanie del corpo e della persona del sovrano. Sicché concepiscono un abbigliamento che, in ragione dei materiali adoperati e dei dettagli preziosi, deve permettere ai sottoposti e prima ancora al gruppo dei pari di visualizzare la sua potenza e il relativo carisma. Il ricorso ad un corredo di simboli più antico della propria esperienza, come quello della Basileia, costituisce non solo un modello facilmente riconoscibile sia dai sottoposti, sia dal gruppo dei pari, ma può fungere quale sorta di "clausola di salvaguardia” per la propria politica di autorappresentazione. La formula che dimostra l'esercizio da parte degli Altavilla di un potere non derivato, viene liquidata come un'usurpazione, che attira il biasimo di quei medesimi pari entro cui il re siciliano auspica di essere integrato (Delogu, 1983; Cantarella, 1996; Cantarella, I998; Cantarella, 200I). Eppure, le aspirazioni autarchiche dei sovrani di Sicilia devono passare necessariamente per la veste ostentata. L'attualizzarsi della promotio regia difatti implica l'obbligo d'investire in abiti ricercati, il cui potenziale affabulatorio viene orientato alla spettacolarizzazione durante le epifanie regie. Un corredo solenne che le fonti denominano vestimenta regalia e distinguono dai vestimenta leviora, adoperati tutti i giorni dal sovrano. I vestimenta regalia sopravvivono presso la Schatzkammer di Vienna, rifunzionalizzati quali insegne del Sacro Romano Impero da Federico II (Elze, 1995). Esse solo di primo acchito sembrano differenziarsi dall'abbigliamento delle rappresentazioni monumentali di Palermo e Monreale, ma ad una più attenta analisi la realtà risulta ben diversa, poiché si riscontra il perpetuarsi almeno a guisa di onde mnemoniche della foggia romano orientale.

La dalmatica regia, denominata "Tonacella», quale fastoso camice di seta, reinterpreta la fenomenologia della porpora, avvicinando la tinta ad un luminoso indaco. Una foggia che si concede la velleità dei «tagli ricurvi» per evidenziare la vita (Tramontana, I993: 86-98; Bauer, 2006a). Le bordature delle maniche sono poi impreziosite con seta rosso granato, ricami in filo d'oro, perle e piastrine decorate con losanghe, di cui ne sopravvivono ben 24 . Tuttavia, la tunica a tinta unita si contrappone a quella rappresentata nel perduto mosaico della cattedrale di Gerace, che vede Ruggero II vestire un abito aureis liliis circumfuso (Pasqua, O., Vitae episcoporum Ecclesiae Hieracensis, 249; Vagnoni, 20I2; Vagnoni, 20I7; Vagnoni, 20I9). Un decoro che trova riscontro nel mosaico della Martorana, laddove la tunica è puntellata con flour de lis (Kantorowicz, I950). I motivi stilistici possono collocare la veste entro l'ampio arco temporale in cui regna Ruggero. Tuttavia gli smalti delle piastrine ne dilatano la cronologia, permettendo una datazione anche più tarda. La resa calligrafica li avvicina piuttosto agli smalti della spada della fine dell'XI sec.; ciò non esclude però un'aggiunta a posteriori (Lipinsky, 1973).

La tonacella viene solitamente indossata su una sottoveste, l'alba, un camice di colore bianco, ben visibile nei mosaici di Monreale al di sotto della dalmatica regia. Questo camice è caratterizzato da un taglio «destrutturato e largo» (Tramontana, 1993: 86-92; Bauer, 2006b). L'abito può essere datato in ragione delle iscrizioni in caratteri arabi collocate su strisce di lino e allocate sotto i galloni a decoro. Queste documentano l'anno II8I. Una data che è però indicativa. Si limita a riferire l'anno di produzione del gallone dell'alba, ma non dell'intero abito, poiché per esso sembrano rifunzionalizzarsi diversi pezzi di tessuto (Al Samman, 1982; Johns, 2006). Dall'iscrizione emerge il nome dei ricamatori: Marzuq, Ali e Mahumad o Mushin, del sarto Tumās e del supervisore Damiyān (Bauer, 1995). Si dimostra così la presenza di lavoratori cristiani e musulmani presso il regio ergasterion (Al Samman, 1982). Il camice bianco sostituisce da un certo punto in poi una veste color porpora, probabile manifattura bizantina, che sopravvive nel corpetto in sciamito posto al di sotto dell'alba. La veste candida è impreziosita da una sorta di pettorale che sul lato anteriore giunge fino al diaframma, mentre sul posteriore si ferma all'altezza delle scapole, decorato con preziosi, fra cui una serie di gemme in modesti castoni infisse in epoca successiva. La texture della veste mostra l'uso di lamè in filo d'oro e di fili tinti in porpora di Tiro nella sola parte visibile, mentre il resto è colorato con tinture più economiche a base di noci di galla e sommacco siciliano con mor- 
dente a base di ferro. Il filame viene adoperato per realizzare una serie di motivi decorativi come quello dell'Arbor Vitae.

Dall'anamnesi dei vestimenta regalia si può dedurre un effettivo distacco dalla mise del basileus ed una riproposizione dell'abbigliamento degli imperatori occidentali? Pare poco probabile. I documenti visuali prodotti dalla corte, non solo rappresentano l'immagine che il re vuole dare di sé, ovvero quella di emulo del basileus, ma lo rendono immediatamente riconoscibile. Costituisce difatti un nonsense adoperare una formula rappresentativa, che poi le concrete epifanie regie smentiscono causando spaesamento visivo nei fruitori. L'iconografia ha altresì a contraddire il contenuto della concessione papale, la quale fissa le insegne del regno? Il privilegio pontificio è del II49 e si inserisce in un quadro di autorappresentazione già formato $\mathrm{e}$ si limita a prendere atto per la maggior parte dei casi dell'uso di determinate insegne, tutte ritrovabili nei documenti visuali della propaganda siciliana. Indi per cui aggiunge poco o nulla. Pertanto, appare plausibile che anche in pubblico il re siciliano abbia ad indossare un abbigliamento se non aderente in toto, almeno riconducibile nelle forme mnemoniche, al corredo del potere romano orientale. Di conseguenza si può presumere che non vi deve essere alcuna dicotomia significativa fra le rappresentazioni iscritte nei documenti visuali e la concreta mise del sovrano locale. Gli ordines coronationis invece sono molto più tardi, riferiti ad un sovrano di nome Federico, forse il futuro imperatore o forse Federico II, figlio di Beatrice Lanza. Risalgono, difatti, ad un'epoca in cui la memoria bizantina progressivamente si attenua, mentre il rito locale si modella sul canovaccio dell'ordo di Magonza, quindi poco possono dire circa la cosuetudine vestiaria del sec. XII.?

La concessione pontificia del II49 menziona il pallium, che nella sua accezione medioevale indica una mantella semicircolare assimilabile al piviale ecclesiastico, sicché il cerimoniale locale di ascesa recepisce il rito d'immantatio. Il manto pervenutoci è decorato da un'epigrafe in

\footnotetext{
9 Elze retrodata gli ordines ad epoca normanna, cfr. Elze, 1973. Per l'ordo di Magonza, cfr. Zug Tucci, 2002.
}

caratteri cufici, che si distende per circa 3 metri sul suo orlo, e fornisce una data precisa ed il luogo della sua produzione. ${ }^{10}$ Una dedica atipica rispetto alle iscrizioni islamiche coeve, perché manca l'invocazione a Dio ed il nome del committente, ma indica solo la «regia officina» o «magazzino» (Tronzo, 2020; Dolezalek, 2017). L'anno 528 corrisponde poi al II33-II34 del calendario giuliano ed, indi, la produzione dell'indumento va a collocarsi nel quarto anno successivo all'incoronazione di Ruggero II. Il complesso programma iconografico riportato sul manto funge da evidenziatore visuale e non si limita a suggerire una manifattura araba, ma evoca piuttosto la tradizione occidentale dei manti imperiali, mentre gli stilemi rimandano ad un "relitto" iconografico dell'oriente antico. Si precisa che manca un diretto paragone nelle evidenze prodotte dal coevo mondo musulmano, poiché i documenti tessili superstiti presentano tessuti a tinta unita su cui vengono applicate bande con iscrizioni (Bauer, 2006b; Tronzo, 2006). Luso del chermes poi non costituisce un punto di rottura con il tradizionale uso della porpora di bizantina memoria, poiché il Medioevo non conosce una precisa lessematica per le diverse sfumature dei colori. Il chermes può essere così ricondotto, come anche l'indaco della Tonacella, alla porpora. Una tinta che può essere considerata regia, giacché evoca il carbunculus, la pietra che identifica la tribù di Giuda; un colore dal sentire messianico dunque. Il tessuto adoperato, un monocromo in seta, potrebbe esser importato, prodotto a Tebe e poi tinto in loco. L'ipotesi evoca così lo scarso successo della produzione serica palermitana. Il decoro poi mette in scena due vigorosi leoni che sopraffanno dei cammelli innanzi ad una palma da datteri, simbolo dell'albero della vita gravido di frutti, ciò conferisce alla lotta un carattere cosmico (Lipinsky, 1973). Un'allusione al ruolo del sovrano quale axis mundi, ma anche un rimando alla Chiesa legittimante. Una simmetricità

\footnotetext{
${ }^{10}$ «Eseguito nel tiraz reale di Palermo dove la felicità e l'onore, il benessere e la perfezione, il merito e l'eccellenza hanno loro dimora; di grandi liberalità, d' un alto splendore, della reputazione, delle speranze; possano i giorni e le notti ivi scorrere nel piacere senza fine né mutamento nell' onore, la fedeltà, l'attività diligente, la felicità e la lunga prosperità, la sottomissione e il lavoro che conviene. Nella capitale della Sicilia, l'anno 528" (trad. it. F. Gabrieli).
} 
nel decoro che è forse tutta occidentale e si oppone al motivo antichizzante e mediorientale. La lettura allegorica dell'immagine evoca il trionfo delle genti normanne, rappresentate dal leone, emblema araldico degli Altavilla, sui cammelli, figura degli arabi. Tuttavia, un proverbio arabo potrebbe spiegare il senso della rappresentazone, giacché riferisce un improbabile costume dei leoni, i quali sarebbero soliti pregare Dio prima di avventarsi sui cammelli per ottenere il vigore necessario (Bauer, 1995). L'estrema stilizzazione dell'anatomia degli animali ed i motivi ricercati come le rosette rimandano poi alle sete bizantine. Nondimeno, le palmette e le stelle iscritte appaiono più tipiche dei tessuti persiani. La rappresentazione del cammello è invece molto rara, l'unica evidenza conosciuta che la riporta si ritrova in un rilievo della facciata della basilica barese di S. Nicola. L'evocazione del Medioriente antico non può però negare quell'aulica citazione che ascrive la formula decorativa ad un sottoprodotto della regalità bizantina. Il locus della bestia feroce che sopraffà un altro animale, ponendo la zampa al collo, si ritrova ad ornamento dell'atto di matrimonio di Teofano con Ottone II, conservato in Wolfenbüttel. In questo caso l'animale dominante è però un grifone, che si impone su un cervo. Il motivo si ascrive ad un repertorio della regalità comune, costituito da formule rappresentative neutre, che appaiono sempre adattabili al contesto d'uso. Le varianti si apprezzano perciò come evoluzioni della formula, che l'arricchiscono di nuovi significanti per rispondere alle esigenze comunicative (Bauer, 1995). Le due borchie quadrilobate con funzione di fibula in sua pertinenza, decorate con disegni geometrici in smalto cloisonné, riportano delle stelle ad otto punte, che evocano altresì sia l'arte copta, sia l'oreficeria islamica del XI sec.. Eppure, la tecnica di manifattura rimanda piuttosto a produzioni costantinopolitane, ma non esclude l'opera di artisti locali. Orbene, l'imaginerie del potere normanno si nutre di diverse tradizioni, che vengono sistematizzate in un contesto dopotutto unitario e volto ad incidere l'immaginario comune.

La fattura del fermaglio del piviale suggerisce poi un rifacimento, tanto che, quasi unanimemente, lo si ritiene non originale. Appare perciò dibattuta l'ef- fettiva modalità di vestizione. È difficile dire se questo paludamento venga indossato a modo di clamide e indi ruotato di $90^{\circ}$ o come mero piviale. La rigorosa simmetricità del disegno e l'esigenza di ottimizzarne la visibilità del decoro, lasciano pensare che questo venga appuntato sul petto; una congettura che si basa su meri indicatori e non convince del tutto. Sappiamo poi che vestire il manto a modo di piviale è diritto esclusivo dell'imperatore d'Occidente, malgrado ciò tale costume non sembra ostacolare più di tanto una qualsivoglia imitatio. Tuttavia i documenti visuali sconfessano l'ipotesi, allorché attestano solo l'uso della chlamys. A riprova di quanto asserito, il follaro emesso da Ruggero II in Salerno dal iı27 al II30 mostra una mera clamide. La rappresentazione di Ruggero intronizzato, posta a corredo del diploma destinato al monastero di Casauria e datato al 27 agosto II4O, conferma altresì l'uso della clamide. L'immagine è leggibile in una copia dell'atto di donazione, che è riportata al $\mathrm{f}$. 248 r del Chronicon Casauriensis; l'originale diversamente è andato perduto. Qui la clamide scende con ampio drappeggio dalla spalla destra, laddove è fermata da una piccola spilla rotonda, mentre una lacinia a motivo vegetale stilizzato percorre tutto il manto. La clamide ricompare ancora in una rappresentazione di Guglielmo II iscritta sull'ottavo capitello della corsia occidentale del chiostro del duomo di Monreale. Il sovrano veste un manto connotato da un taglio di sbiego, che disegna un angolo acuto. Tale morfologia rimanda alla foggia del paludamento rappresentato nel codice Coislin 79 al f. 2 r e al f. 2 v, che rappresenta Niceforo Botaniate, ora conservato presso la Bibliothèque nationale de France. Una tipologia sartoriale condivisa pure dal manto rivestito da una scultura rappresentante Carlo Magno presso la chiesa di Müstair. A questo si aggiunge un documento più tardo: il Liber ad honorem Augusti. Al f. 3 le scene II e III rappresentano Ruggero II a cavallo con una clamide resa con un pallido arancione, che forse traduce il chermes. Si raffronta un potente indicatore dell'uso esclusivo della clamide e di una precisa cromia per identificare il re.

Occorre altresì considerare l'effettività della presenza del loros nel corredo dei vestimenta regalia. L'insegna 
compare nei documenti visuali, ma non viene mai citata nei più tardi ordines $\mathrm{A}$ e $\mathrm{B}$, cosa che non deve meravigliare, poiché è indossata prevalentemente il giorno di Pasqua e non nei cerimoniali di ascesa. Nemmeno compare nella concessione papale del II49. Eppure, la ricca stola della Schatzkammer di Vienna, prodotta nel Nord Italia nel sec. XIV, conferma l'esistenza di un più antico capo (ora andato perduto) in uso da parte degli Altavilla ed assimilabile all'insegna del basileus (Bauer, 2006a). Tale esistenza sembra confermata da due inventari delle insegne imperiali, che testimoniano la presenza di più stole assimilabili al loros. Il I7 di settembre del I246 Corrado IV (I228-I254 d.C.) entra in possesso delle insegne imperiali conservate nel castello di Trifels, consegnate dalla contessa Isabella, moglie del siniscalco Philipp von Falkenstein. L'inventario menziona ben tre cinture di seta, fra cui si possono intravedere addirittura due loroi, poiché il cingulum è solo uno. L'inventario del 1359 diversamente attesta la consegna delle imperialia insignia a Lodovico, figlio di Carlo IV (I3I6-I378 d.C.), da parte del marchese Lodovico di Brandeburgo. Ivi si afferma la presenza di ben due loroi: il primo con pietre preziose e perle (forse la cosiddetta stola delle aquile) ed un'altra stola andata persa, priva di pietre, presumibilmente un antico loros siciliano (Schwinger, 2006). Nondimeno l'utilizzo del loros nel corredo regale siciliano viene dimostrato dalla foggia della stola delle aquile, la quale probabilmente sostituisce un equivalente di epoca normanna. La sua lunghezza di sei metri, eccessiva per una normale stola e poco agevole per la vestizione a modo liturgico, già fa pensare ad un loros. Difatti i documenti iconografici dimostrano come venga goffamente avvolta sul petto ad X. Altresì, un altro indizio viene dalla collocazione delle placchette decorate con aquile, che stranamente cambiano disposizione lungo l'ampia metratura dell'accessorio. Sicché, se questa viene rivestita modellandola secondo la foggia ad Y, come nel mosaico rappresentante Ruggero II alla Martorana, tutte le aquile vengono a trovarsi in piedi. Un'ipotesi rafforzata dalle cuciture della stessa, che si trovano nei punti da ripiegare per assecondare la fisionomia del corpo. L'imitatio By$z$ antii normanna allora sembra colonizzare il vestiario degli imperatori d'Occidente, che non solo utilizzano i loroi siciliani, ma addirittura quando uno dei due si ha a logorare, ne commissionano uno nuovo che lo sostituisce. La mutuazione del loros da parte degli Altavilla è giustificata pure sul piano ideologico, poiché rende il sovrano locale una figura Christi, imitando la christomimesis inscenata dal basileus nella processione pasquale a Costantinopoli (de caer. I, 37; Constitutum Constantini 257). Un incunabolo che non sfugge a Filagato da Cerami, il quale nell'omelia per la Domenica delle Palme può evocare le suggestioni cristiche: $\dot{e}$ al tempo stesso una festa di Dio e del sovrano... (Filagato da Cerami, Omelia XXVII). La panoplia della dottrina del potere romano orientale ha ad avvallare le velleità autocratiche normanne, attraverso l'enfatizzazione del diretto rapporto tra Dio ed il sovrano, mediato da Cristo, a cui il re locale si assimila a tramite di questo medio atto a renderne visualizzabile il carisma ( Vagnoni, 20II). Tale soluzione si dimostra indispensabile per rafforzare la cognizione da opporre al gruppo dei pari. Un uso comprovato dalla presenza massiccia del loros nei documenti visuali del Regno normanno, che viene tradotto in due diverse foggie. E se sul piano numismatico i referenti immediati sono i folles salernitani del longobardo Gisulfo II (Grierson, 1959), che si presenta rivestito del loros e recupera l'iconografia di un Histamenon Nomisma aureo di Costantino IX, il follis salernitano di Roberto il Guiscardo è frutto della ribattitura dei conii in rame emessi da Romano I e Costantino IX. Il follis di Ruggero II datato tra il II27 ed il IIzo presenta poi il loros del tipo ad Y con ampia falda. Invece il follis emesso con buona probabilità dalla zecca di Messina dal iızo mostra il loros-scapolare di moda sotto i Comneni (Travaini, I973: 5I; 246; 283; tav. I2, n. I9I; Engel, I882: 32-5I; Vagnoni, 20I2). Una foggia, quella a scapolare, che ricompare nella bulla ruggeriana in piombo conservata ad Ariano Irpino. E se le immagini ufficiali descrivono con cura calligrafica le gioie e gli altri ornamenti caratterizzanti l'insegna, il ricco loros a scapolare che compare nei ducali emessi a seguito della riforma monetaria del II4O non presenta la stessa attenzione al dettaglio. Questa formula descrittiva viene ripresa pedissequamente da Guglielmo 
I nei cosiddetti «ducales novi» (Travaini, 1973: 68; 220222; Grierson, 1973; Fitzgerald, 2020; Vagnoni, 2012). La bulla plumbea di Guglielmo II, conservata al Tabulario di Santa Maria la Nova in Monreale, brilla altresì per resa calligrafica e mostra un ricco loros a scapolare. La medesima cura si evince dal sigillo di Guglielmo II, che presenta però un loros ad $\mathrm{Y}$, a dimostrazione dell'intercambiabilità del locus iconografico (Vagnoni, 20I2; Scirea, 2020). Il loros ad Y appare infine in un'altra immagine monumentale a mosaico presente nel duomo di Monreale, laddove Guglielmo II offre alla Vergine la reductio della chiesa (Kantorowicz, I960; Salmaso, 2019).

\section{UN DETTAGLIO D'ECCELLENZA PER IMMAGINARE}

E VISUALIZZARE IL CARISMA DEL RE: IL SEPOLCRO

DI PORFIDO E LA MONUMENTALIZZAZIONE DELLO SPAZIO FUNERARIO

Lattività di immaginazione degli intellettuali, concretizzatasi in strategie di rappresentazione che permettono di visualizzare il carisma connesso ad un potere originario e derivante direttamente da Dio, trova il suo culmine nell'appropriazione del porfido, quale dettaglio d'opulenza ed appannaggio simbolico esclusivo della maestà imperiale, nonché corollario della «liturgia della porpora» (Cavallo, 1996: I49; Carile, 2002). Il porfido costituisce un lemma di un discorso complesso, che riflette i valori della monarchia e si esprime attraverso i simboli. Esso è forse il vero protagonista dei «sistemi rappresentativi» proposti al gruppo dei pari, poiché dichiara l'esercizio di prerogative giuridiche, che vanno ben oltre quelle proprie di un re (Catalano, 1965/1966: I79-190). La cesura causata dalla morte del sovrano impone l'assolutizzazione del corredo simbolico della monarchia attraverso il ricorso ad un segno indiscusso d'eccellenza da destinare ai monumenti funebri regi. Il segno propone una connessione intenzionale tra marmo e sovrano, mentre suscita la visualizzazione della sua vita ultraterrena, giacché ha finalità "protettive» (Tronzo, 20II: 20-2I). La funzione meramente visuale si realizza nell'identificazione fra contenitore e contenuto, tanto da costituire un unicum all'occhio. La mansione protettiva si consuma nella destinazione della pietra a scrigno del "mistero della regalità", perché monumentalizza lo spazio destinato alla morte ed isola l'iniziato dalle impurità, garantendo la trascendenza dell'istituzione. Tale mutuazione si afferisce al precedente costantinopolitano che utilizza arche tratte dal porfido per l'inumazione dell'imperatore fino al seppellimento di Marciano. Questa pietra viene reperita dalle cave del Mons Porphyrites presso Djebel Dukhan, che sappiamo essere nel sec. X oramai esaurite. Gli utilizzi successivi sicuramente costituiscono reimpiego, rifunzionalizzazione di spolia o riutilizzo di giacenze. Un materiale che si presume reperito a Roma, probabilmente sotto forma di colonne da rielaborare. La scelta del porfido poi potrebbe essere connessa al rapporto dialettico col papato, allorché i pontefici operano il riuso politicamente orientato di tombe di porfido come quella di S. Elena o della "conca porfirea" collocata innanzi al Pantheon e appartenuta al mausoleo di Adriano (Herklotz, I985; Herklotz, 1994). Gli Altavilla, loro vassalli, non possono essere certo da meno. Eppure non si può trascurare l'intervento dei consiglieri greci che possono aver suggerito l'uso del porfido quale forma di imitatio Byzantii. Qualsivoglia sia la sua fonte di ispirazione, la Roma dei papi o la Bisanzio dei basileis, il suo ricorso può liquidarsi quale «bizantinismo» dei bizantini», giacché ha a trattarsi sempre di "bizantinismo" di marca occidentale», che «non per questo cessa di essere influenza bizantina» (Pertusi, 1964: 226). Il sepolcro, quale mobile che contiene il sovrano, configura un fenomeno architettonico ed esalta sul piano visuale il rappresentante dell'istituzione deceduto. Pertanto può essere ritenuto l'insegna più duratura del potere ed evoca il locus del doppio corpo del sovrano (Kantorowicz, 1989). Questi si presenta vivo, seppur il suo corpo riposa nel sepolcro, giacché vive ancora come corpo "pubblico". Dalla sede d'eccellenza del porfido il trapassato ostenta la pienezza del suo potere, obbigando al timor reverentiae. Questo può collocarsi in uno spazio potenziato da un altro indicatore visuale come il baldacchino, cosa che evoca la copertura in porfido ad ornamento del trono presso il Consistorium del Sacro Palazzo; ciò lo rende 
un sottoprodotto della bizantinità. Eppure, può essere ricondotto ad una più generica imitatio imperii, stante l'onnipresenza del baldacchino nelle rappresentazioni della majestas dell'Impero d'Occidente. Una presenza che rimanda comunque ad un "bizantinismo" accolto dagli occidentali. Le sei colonne che connotano i baldacchini delle tombe degli Altavilla, oltre le ovvie ragioni di statica, si afferiscono ad una scelta estetica. Il più autorevole precedente può ritrovarsi nel sepolcro di Goffredo di Buglione presso la Cappella del Calvario in Gerusalemme, il quale è ora apprezzabile solo attraverso un disegno al f. I03 del Ms.Latino 9233, conservato presso la Libreria Vaticana. Un prototipo consistente in un baldacchino con sei colonne ed una copertura cosiddetta a "libretto". L'evocazione del modello sembra attualizzare la pretesa normanna verso il trono di Gerusalemme in ragione dei diritti che Ruggero vanta a causa del matrimonio di Adelasia del Vasto, sua madre, la quale in seconde nozze diviene regina di Gerusalemme. Costoro scelgono così di connettersi direttamente alla memoria dell'Advocatus Sancti Sepulchri, che però re non è mai stato.

L'utilizzo del porfido per la monumentalizzazione degli spazi funerari attecchisce entro il programma automagnificatorio pensato da Ruggero per il duomo di Cefalù, destinato a mausoleo dinastico dal II45. Un'aula che costituisce il memoriale della fondazione del Regno ed accoglie la sua tomba monumentale (Delogu, 1995; Delogu, 1998). Deve stigmatizzarsi come l'abuso del porfido da parte di Ruggero si consuma in un periodo in cui il Regno appare debole sul piano internazionale, stretto fra la pressione dei due imperi e del papato che rivendica per sé il Meridione d'Italia, considerandolo feudo del Patrimonio di S. Pietro. Il diploma di destinazione dell'edificio a mausoleo, un documento solitamente ritenuto un falso e forse risalente al regno dei due Guglielmi, illustra la significatività dello status symbol. Tuttavia, dal testo non si evince se a quella data i sepolcri forniti di baldacchino fossero già in situ, collocati con tutta probabilità fra le braccia del transetto e presso il coro dei canonici (Valenziano y Valenziano, 1979). La tomba del sovrano sembra essere collocata a nord, «in cornu Evangelii», mentre l'altro sepolcro, identico e vuoto, viene allocato «in cornu epistolae» a sud. In Sicilia sopravvive così la tradizione tutta normanna dei sepolcri «intra chorum et altare» (La Duca, 2002: 304; Molina Lopèz, 20I4: 56-64). I sepolcri probabilmente vengono lavorati dall'opificio della cappella palatina palermitana, per poi essere montati già finiti a Cefalù. Federico II nella sua ridefinizione delle politiche di autorappresentazione nel I209 trasla i due sepolcri nella cattedrale di Palermo. ${ }^{11}$ Una scelta non indolore, che viene pagata con la scomunica da parte del vescovo cefaludense. Scomunica revocata solo a seguito della donazione a favore della diocesi del feudo di Coltura, attestata in un diploma del settembre I2I5 (Andaloro, 2002). Per la loro costruzione si ipotizza che siano state adoperate due colonne, una sicuramente di notevoli dimensioni, con ampia circonferenza ipotetica, ed una forse più piccola. La più grande viene sezionata in due tronconi, forse ad un terzo della circonferenza, per ricavare entrambe le casse. Da quella più piccola, sezionata in linea verticale, invece vengono tratti i coperchi. Ipotesi plausibile in ragione del fatto che la colonna usata per le casse, per quanto grande, non può fornire adeguato materiale di risulta per i coperchi (Gandolfo, 2006). Si è pensato che in origine Ruggero II abbia previsto due sarcofagi sostanzialmente identici e posti in simmetria davanti al presbiterio. Cosa che non spiega la maggiore ricchezza del sarcofago occupato poi da Federico II rispetto a quello di Enrico VI. Il più ricco apparato iconografico delle mensole zoomorfe e del coperchio con spioventi decorati da clipei potrebbe essere ricondotto ad un programma voluto da Federico, che rimodula l'evidenza (Barolo, 2002; Vergara Caffarelli, 2002). Una tesi che però non convince. Più segnatamente, il sarcofago occupato da Federico II presenta la forma di arca, mentre

\footnotetext{
${ }^{11}$ Le salme dei duchi di Sicilia sono custodite presso la cappella di Santa Maria Maddalena del duomo di Palermo. Questa viene distrutta dall'arcivescovo Walter de Mill durante i lavori di rimodernamento del duomo. Fra il II84 e il II87 si sceglie per i sepolcri una collocazione a meridione ed a sinistra dell'altare. Cfr. Barolo, 2002. Un disegno a penna acquerellata proveniente dal Teatro Geografico Antiguo y Moderno del Reyno de Sicilia, un manoscritto del 1686 ora alla Biblioteca Nacional de Madrid, li mostra presso la cosiddetta Cappella del SS. Sacramento, cfr. La Duca, 2002. Durante i lavori del I78 le tombe vengono collocate presso la campata della navata laterale destra in propiscenza dell'ingresso meridionale.
} 
il suo coperchio a spiovente è dotato su entrambi i lati di una sequenza di tre clipei: compaiono il Cristo Pantocratore da un lato e l'Odeghitria dall'altro, disposti fra i simboli degli evangelisti. Ai lati del coperchio viene ancora rappresentata la bügelkrone, col doppio arco ad ogiva. La cassa del sepolcro presenta poi un elegante fregio di foglie d'acanto. Un lato della stessa è ornato con una croce greca patente, mentre l'altro presenta un battente con testa leonina. Le mensole zoomorfe mostrano dei leoni accovacciati, con le code intrecciate ed annodate al lato. Un animale totem dunque, che assume la funzione di guardiano dei sepolcri ed allorché trattiene fra le zampe figure antropomorfe o zoomorfe si dimostra piuttosto quale fiera espressione del diritto dei governanti all'esercizio della giusta violenza. Il sepolcro è poi collocato sotto un baldacchino di porfido sorretto da colonne, che viene probabilmente danneggiato durante il trasporto a Palermo ed integrato con inserti in granito. La durezza del materiale impone ai lapicidi di ripiegarsi sulle foglie d'acqua per i capitelli che interpretano lo stile corinzio. La morfologia lanceolata del vegetale non richiede particolare attenzione per i dettagli interni. L'architrave è invece ornata da maschere antropomorfe. La sinteticità dei tratti, quale soluzione di compromesso con la durezza del materiale, permette appena di indicare l'età dei rappresentati. Le capriate sempre in porfido sono ornate da due colonnette tortili per lato e sorreggono una copertura a libretto in lastre di granito. Queste integrazioni dimostrano che al momento della ridefinizione è impossibile anche per un imperatore poter reperire sul mercato del porfido. Orbene, per ragioni stilistiche la decorazione deve essere additata alle maestranze specializzate gravitanti attorno alla corte, che hanno scolpito il candelabro pasquale della cappella palatina. Le affinità formali permettono di accogliere una datazione intorno al II54, anno della morte di Ruggero, o fors'anche di qualche anno successiva, ma poco sembrano avere a che fare con la cifra stilistica della plastica proposta sotto Federico. ${ }^{12}$ La scarsa compatibilità è così

\footnotetext{
${ }^{12}$ La datazione precoce si basa su dati stilistici e su analogie formali nella resa dei volti e delle anatomie animali che si riscontrano fra il sepolcro ed il candelabro della cappella palatina. Analogie che si spiegano
}

confermata dalle integrazioni in granito e dalla resa raffinata dei dettagli del capitello con aquile, che molto si allontana dalla sommarietà del modellato porfireo.

Diversamente il sarcofago occupato da Enrico VI prevede un programma ornamentale che rimanda ad un precedente stilistico autorevolissimo: la conca porfirea di Adriano, ora nella cappella Corsini, fatta trasferire al Laterano da Innocenzo II. La scelta mostra una preferenza per "l'antico", come dimostra il bordo profilato con ovuli. Ciò contrasta con i vezzi della moda, che suggeriscono di optare per la più moderna soluzione del giacente. Il ricorso alla classicità e l'impiego dello status symbol costituiscono dunque un consapevole strumento di legittimazione. Il sepolcro anche in questo caso è caratterizzato da un tetto a spiovente, ma privo di decori. Su uno dei lati corti compare un motivo floreale, assimilabile ad una rosetta ad otto petali, mentre nell'altro si iscrive una corona, che Deér definisce prettamente femminile. Il lato breve del sarcofago presenta un motivo vegetale inserito in una corona. La cassa a sua volta è poggiata al di sopra di una doppia mensola modanata. L'evidenza sul versante plastico vuole rappresentare in modalità molto semplificate il prototipo romano. L'opera dei lapicidi locali è indirizzata a trovare soluzioni di compromesso fra le sinuosità del modello, le caratteristiche del materiale e le proprie capacità tecniche. La notevole riduzione dello spessore dei corpi scanalati e delle volute dei sostegni si inserisce in un percorso che segna la riscoperta della complicata tecnica per lavorare il porfido. Si raffronta nell'acerbità della metodologia di lavoro un ulteriore indicatore atto ad additare una commissione alquanto precoce dell'evidenza monumentale. Il relativo baldacchino è invece rimasto integro e presenta capitelli piuttosto stilizzati, decorati con foglie lanceolate. Le architravi ed il timpano dalle linee sobrie considerano l'unico fasto del porfido.

nell'ambito di una scuola palatina specializzata nelle tecniche di lavorazione del porfido. La mancanza di virtuosismo nella traduzione plastica e l'assenza di profondità nella resa dei piani sono un marchio tecnico della scuola. Questi sono null'altro che espedienti per assecondare la durezza del porfido, dato che le tecnologie antiche di lavorazione sono andate perdute. Cfr. Deér, 1959; Gandolfo, 2006; Vergara Caffarelli, 2002; Molina López, 2014. 
Costanza, a mezzo del suo testamento datato al 25 dicembre del Iı98, palesa il desiderio d'essere seppellita presso le tombe del padre Ruggero II e del marito Enrico VI, ubicate nella Cattedrale di Palermo. Per questa si progetta, forse su commissione di Federico, un sepolcro in porfido. Il modello da seguire è quello del sepolcro di Enrico VI. La qualità della fattura sembra documentare che probabilmente in quel momento si ha a disposizione una sezione di colonna, forse pari ad un terzo. I lapicidi riescono a ricavare da un pezzo unico il fondo della cassa sul cui lato corto iscrivono una ghirlanda contenente una croce greca patente. Procedono al montaggio del coperchio e delle mensole traendoli da lastre frutto di un'opportuna sezione del materiale restante. Il coperchio in particolare risulta dal montaggio di diversi pezzi e come nelle tombe alla cappuccina lo spiovente è composto da lastre inclinate ed appoggiate l'una all'altra. Una delle cuspidi poi accoglie il rilievo di una bügelkrone, attributo maschile per Deér, che ha fatto presupporre un'errata ricomposizione dei corpi di Costanza ed Enrico dopo una qualche frettolosa ispezione (Deér, 1959). Il programma decorativo poi viene rimodulato in ragione della poca pietra posseduta e si caratterizza per le scanalature poco profonde delle mensole e la resa sommaria del fregio ad ovuli. L'indisponibilità del porfido costringe ad adoperare per il baldacchino a libretto il marmo bianco decorato con mosaici, mentre i capitelli sono ornati da foglie d'acanto. Si è persino ipotizzato il riuso di una tomba già esistente a destinazione maschile, data la corona. Forse quella sita nella cappella palatina e destinata a Guglielmo I (commissionata prima del II83?) o quella occupata da Tancredi (prodotta prima del II94?), da cui il marito Enrico VI ha fatto sottrarre le insegne regali. Tuttavia il tentativo mediocre d'imitazione non offre dettagli utili a delineare una cronologia specifica.

Ben più complesse sono le vicende che concernono la sepoltura di Ruggero II. L'arcivescovo Walter de Mill alla morte del re, il 26 febbraio II54, fa collocare la salma in Palermo perché la chiesa di Cefalù non è ancora consacrata. Sicché può assicurare alla propria sede un ruolo primaziale sull'intero Regno. Si prevede per il sovrano un'urna provvisoria che sembra essere utilizzata fino al II70, delle cui fattezze si sa poco o nulla. Dopo quella data l'arcivescovo Walter de Mill commissiona la riedificazione della cattedrale. Durante questo periodo si ignora l'eventuale collocazione delle spoglie. Si può però ipotizzare che nel Iı87 il sepolcro sia già deposto nel transetto meridionale, perché un'iscrizione del 1185 sita nell'abside ci informa che il corpo è già ultimato. La salma viene così deposta in un'arca dalle forme lineari, che ricorda un reliquiario limosino, sobrio e quasi minimale, in cui l'unico fasto è dato dal porfido con cui viene costruito. Per la tomba di Ruggero II l'ambizioso vescovo committente si scontra non solo col problema della carenza del porfido, ma anche con gli ingenti costi del materiale e le difficoltà di appannaggio per un privato. Un tale uso deve risarcire la volontà testamentaria di Ruggero già disattesa ed opporsi al capitolo cefaludense che, in virtù dei sepolcri posseduti, reclama l'esecuzione del testamento. L'urna è creata dalla mera connessione di lastre di porfido, tratte dalla sezione di un blocco non troppo grande e forse di risulta. Gli ingenti costi che il vescovo deve sobbarcarsi obbligano poi ad optare per un progetto diverso da quello adoperato per le tombe di Cefalù. Lunica velleità plastica che viene concessa è quella dei quattro telamoni in marmo bianco, che fungono da mensola per la cassa. I telamoni a loro volta poggiano su un basamento rivestito in lumachella grigia, proveniente da Cefalù. Il baldacchino in marmo bianco, con capitelli corinzi ed inserti in mosaico, funge da contrappunto a quello di Costanza (Rossi Vairo, 20r8).

Guglielmo II altresì concepisce un complesso monumentale che prevede in continuità la chiesa dinastica, il convento dei benedettini e la sede regia, laddove prevede oltre alla propria tomba, che si colloca sotto l'altare maggiore a guisa di un santo, anche quella del proprio padre. Per Guglielmo I si utilizza il porfido e si segue ancora una volta il modello cefaludense. Larca è contraddistinta da un coperchio a spiovente con croci palmate poste nei profili cuspidati, mentre la cassa è decorata nei lati corti da due ghirlande con inscritta una foglia di edera. Il bordo è invece caratterizzato dal solito profilo ad ovuli. Il sarcofago a sua volta è posto su delle doppie mensole, ornate da modanature. E se per la 
resa della cassa in un sol pezzo si può ipotizzare l'uso di una colonna di dimensione rilevante, si osserva come il coperchio sia realizzato in due tronconi, probabilmente con materiale di risulta; lo stesso accade per le mensole, come dimostra la loro forma asimmetrica. Il sepolcro in origine è fornito di un baldacchino andato perso nell'incendio del I8II, che ora costituisce materiale erratico collocato nel chiostro annesso.

\section{CONCLUSIONE}

L'indagine concernente le implicazioni dell'attività d'immaginazione degli intellettuali della corte normanna di Sicilia ha sufficentemente dimostrato come la cultura materiale della regalità venga pensata per offrire una serie di soluzioni adeguate a visualizzare il carisma posseduto dal re locale. La volontà di rendere effettivamente visibile una tale elaborazione si orienta così verso le cosidette strategie di opulenza, che sono poste in essere dalla casa di Altavilla e si indirizzano non solo a suggestionare l'avventore con un dispendio di risorse che crea un clima favolistico attorno alla corte, ma vogliono dimostrare l'esercizio di un potere originario, derivante direttamente da Dio. La volontà di ottimizzare e rendere più performante la visualizzazione del carisma del sovrano è attualizzata a medio di una serie di emblemi che suscitano la deferenza nei sottoposti e devono persuadere il gruppo dei pari. La monarchia attraverso il ricorso alla formula del «a Deo coronatus", che si riscontra nella produzione visuale e nella retorica, offre non solo una soluzione adeguata a rendere visibili le elaborazioni dell'immaginazione degli intellettuali, ma propone un modello condiviso e comunque gradito. Si ripropongono in Sicilia le soluzioni descrittive del potere del basileus e si tentano di risolvere i diversi gap che l'esperienza locale sembra presentare sia sul piano del diritto, sia sul piano ontologico. Le soluzioni rispondono pure alle esigenze del pubblico locale, perché ripropongono il background di un territorio già parte della Basileia. La veste poi configura un segno sensibilissimo ed atto a rendere meglio percepibile l'eccellenza acquisita. L'abbigliamento orbene permette di visualizzare il potere assoluto che si vuole detenuto da colui che la ostenta, così come viene immaginato dagli intellettuali di corte o forse anche meglio. Il rango difatti obbliga ad un corredo vestiario adeguato, nonché a fornire ai fruitori elementi utili alla propria legittimazione. L'enfatizzazione degli emblemi del potere rafforza l'istituzione e configura una clausola di "salvaguardia materiale", che "blinda" la monarchia. La scelta del porfido per i sepolcri poi non solo dichiara le velleità imperiali immaginate per gli Altavilla, ma permette la visualizzazione di un potere che perdura persino oltre la morte, mentre avvalla l'identificazione fra contenitore e contenuto, con mansione protettiva. Esso funge altresì da evidenziatore visuale degli spazi concessi dalla propagada regia alla morte e favorisce la monumentalizzazione dell'ambiente funerario. Le soluzioni adoperate per immaginare la monarchia siciliana aprono ad una nuova fase vitale per i loci del potere della Basileia, i quali vengono rifunzionalizzati, risemantizzati ed adeguati nel nuovo contesto in cui attecchiscono allo scopo di visualizzare in modo ottimale i valori della monarchia locale. Queste formule dall'alto valore icastico difatti appaiono davvero funzionali per uno Stato giovane e privo di una tradizione rappresentativa propria come il Regno di Sicilia, nonché avvallano sul piano visuale le esigenze di autarchia, rendendone più performanti i prodotti della cultura materiale. Le formule della Basileia costituiscono insomma dei modelli culturali mutuati volentieri, poiché aiutano gli avventori a visualizzare una serie di elaborazioni immaginate dagli intellettuali di corte per ottimizzare le epifanie del corpo e della persona del sovrano. Esse, al contempo, soddisfano la domanda sociale e vanno incontro all'aspettativa del pubblico, dichiarando infine la legittimità della novella monarchia. 


\section{BIBLIOGRAFIA}

Al SAmman, T. (1982): «Arabische Inschriften auf Krónungsgewàndern des Heiligen Ròmischen Reiches", Jahrbuch der Kunsthistorischen Sammlungen in Wien, 78, pp. 7-34.

ANDALORO, MARIa (2002): «Per la conoscenza e la conservazione delle tombe reali della Cattedrale di Palermo: linee storiche e storico artistiche», en $I l$ sarcofago dell'imperatore. Studi, ricerche e indagini sulla tomba di Federico II nella Cattedrale di Palermo 1994-I999, Palermo, Regione Siciliana, pp. I35-I 48 .

AndenNA, Giancarlo (2006): «Dalla legittimazione alla sacralizzazione della conquista (I042-II40)», en I caratteri origini della conquista normanna. Diversità e identità nel Mezzogiorno (I030-II30), Atti delle Sedicesime Giornate Normanno-Sveve, Bari, 5-8 ottobre 2004, Bari, Dedalo, pp. 371-405.

Barolo, M. T. (2002): «Federico II 'morto e vivente': significati della tomba nel Duomo di Palermo", Labyrinthos 2I, 4I/42, pp. 3-23.

BRÜHL, C. (1987): Rogerii II. regis diplomata latina, en Codex diplomaticus regni Siciliae, Koln-Wien.

Brühl C., Giunta E., Guillou, A.: (ed.) (1987), Diplomata regum et principum e gente Normannorum, II I, Köln-Wien.

BAuer, Rotraud (2006A): «Il manto di Ruggero II e le vesti regie», en Andaloro, Maria (ed.), Nobiles Officinae: perle, filigrane e trame di seta dal Palazzo Reale di Palermo (Vol. I-2), Catania, Maimone, pp. I7I-I80.

— (2006B): «Le vesti e le insegne per l'incoronazione dei re e degli imperatori del Sacro Romano Impero", en Andaloro, Maria (ed.), Nobiles Officinae: perle, fligrane e trame di seta dal Palazzo Reale di Palermo (Vol. I-2), Catania, Maimone, pp. 425-429.

- (1995): «Il manto di Ruggero II», en D’Onofrio, Mario (ed.), I Normanni popolo d'Europa. 1030-I200, Catalogo della Mostra, Roma, Palazzo Venezia, 28 gennaio-30 aprile 1994, Venezia, Marsilio, pp. 278-287.

Cantarella, Glauco Maria (1996): «Historia non facit saltus? Gli imprevisti normanni», en Cantarella, G.M. y Santi, F. (edd.), I re nudi. Congiure, assassini, tracolli ed altri imprevisti nella storia del potere, Spoleto, Centro Italiano di Studi sull'Alto Medioevo, pp. 9-38.

- (1998): La Sicilia e i Normanni. Le fonti del mito, Bologna, Patron.

- (200I): «Il pallottoliere della regalità: il perfetto re della Sicilia normanna», en Corrao, P. y Mineo, I.E. (edd.), Studi in onore di Vincenzo D'Alessandro, Dentro e fuori la Sicilia, Roma, Viella, pp. 29-44.

CARILe, Rocco Antonio (2002): «Le insegne del potere a Bisanzio», en $L a$ corona e i simboli del potere, Rimini, Il Cerchio-Cantagalli, pp. 65-I24.

Carver, Martin y Molinari, Alessandra (2020): «Sicily and England: Norman Transitions Compa», en Winkler, E.A. et all. (edd.), Designing Norman Sicily. Material Culture and Society, Woodbridge, The Boydell Press, pp. 133-165.

Catalano, Gaetano (1965/1966): «Filippo II e le tombe dei Re di Sicilia», Archivio Storico Siciliano, serie III, I6, pp. I79-I9o.

Cavallo, Guglielmo (1996): «La potenza della porpora», Il Sole 24 Ore, p. I49. DAwn, Marie Hayes (2020): Roger II of Sicily: Family, Faith, and Empire in the Medieval Mediterranean World, Turnhout, Brepols Publishers.

DeÉR, Josef (1959): The Dynastic Porphyry Tombs of the Norman Period in Sicily, Cambridge (Massachusetts), Harvard University Press.

Delle Donne, Fulvio (2006): «Liturgie del potere: le testimonianze letterarie, in. Nascita di un regno», en Poteri signorili, istituzioni feudali estrutture sociali nel Mezzogiorno Normanno (II30- II94), Bari, IO-I3 ottobre 2006, Bari, Dedalo, pp. 33I-368.
Delogu, Paolo (1983): «Idee sulla regalità: l'eredità normanna», en Potere, società e popolo tra età normanna ed età sveva (II89-I2I0), Atti delle Quinte Giornate Normanno-Sveve, Bari-Conversano, 26-28 ottobre 1981, Bari, Dedalo, pp. 185-2I4.

- (1998): «La. committenza degli Altavilla: produzione monumentale e propaganda politica», en en D’Onofrio, Mario (ed.), I Normanni popolo d'Europa. I030-I200, Catalogo della Mostra, Roma, Palazzo Venezia, 28 gennaio-30 aprile 1994, Venezia, Marsilio, pp. I88-192.

Di Cosmo, Antonio Pio (2018): «Pensare la monarchia in Sicilia: i "Motivi erranti della Regalità" costantinopolitani e le "Strategie della Parola” nella propaganda della Corona Normanna», Potestas I2, pp. 5I-8I.

Dolezalek, Isabelle (2017): Arabic Script on Christian Kings. Textile Inscriptions on RoyalGarments from Norman Sicily, Berlin, De Gruyter.

Elze, Reinhard (1973): «Tre Ordines per l'incoronazione di un re e di una regina del regno normanno di Sicilia», en Atti del Congresso Internazionale di Studi sulla Sicilia Normanna, Palermo, 4-8 dicembre 1972, Caltanissetta-Roma, Palermo, Sciascia, pp. 438-459.

- (1995): «Le insegne del potere», en Strumenti, tempi e luoghi di comunicazione nel Mezzogiorno normanno-svevo, Atti delle Undicesime Giornate Normanno-Sveve, Bari, 26-29 ottobre 1993, Bari, Dedalo, pp. I13-I29.

ENGEL, Arthur (1882): Recherches sur la numismatique et la sigillographie des Normands de Sicile et d'Italie, Paris, Ernest Leroux, Paris.

FitZgerald, Liam (2020): «Imperial Iconography on the Silver Ducalis: Cultural Appropriation in the Construction and Consolidation of Norman Royal Power», en Winkler, E.A et all. (edd.), Designing Norman Sicily. Material Culture and Society, Woodbridge, The Boydell Press, pp. II4-I32. GaGÉ, Jean (1933): «La théologie de la Victoire impériale», Revue Historique I7I, pp. I-43.

Gandolfo, Francesco (2006): «Il porfido», en Andaloro, Maria (ed.), Nobiles Officinae: perle, filigrane e trame di seta dal Palazzo Reale di Palermo (Vol. I-2), Catania, Maimone, pp. 425-429.

Grierson, Philiph H. (1959): «The Salernitan coinage of Gisulf II (I052-I077) and Robert Guiscard (1077-1085)», en Papers of British School at Rome, 2, pp. $37-59$.

- (1973): Catalogue of the Byzantine Coins in the Dumbarton Oaks Collection and in the Whittemore Collection, 3 vols., Washington D.C.., Dumbarton Oaks Research Library.

Herklotz, Ingo (1985): “"Sepulcra” e "monumenta” del medioevo", Studi sull'arte sepolcrale in Italia, Collana di studi di storia dell'arte, V, Roma, Nantes.

— (1994): «Lo spazio della morte e lo spazio della sovranità», en D’Onofrio, Mario (ed.), I Normanni popolo d'Europa. 1030-I200, Catalogo della Mostra, Roma, Palazzo Venezia, 28 gennaio-30 aprile 1994, Venezia, Marsilio, pp. 320-326.

Houben, Hubert (1999): Ruggero II di Sicilia. Un sovrano tra Oriente e Occidente, Roma - Bari, La Terza.

JoHns, Jeremy (2006): «Le iscrizioni e le epigrafi in arabo», en Andaloro, Maria (ed.), Nobiles Officinae: perle, filigrane e trame di seta dal Palazzo Reale di Palermo (Vol. I-2), Catania, Maimone, pp. II9-I3I.

Kantorowicz, Ernst (1950): «On the Portrait of Roger II in the Martorana in Palermo», en Proporzioni. Studi di storia dell'arte, III, Firenze, Sansoni, pp. 30-35.

- (1960): I mosaici di Monreale, Palermo, Flaccovio.

- (1976): «The Byzantine Contribution to Western Art of the Twelfth and Thirteenth Centuries», en Kleinbauer, W.E. (ed.), The Art of Byzantium 
and the Medieval West: Selected Studies, Bloomington-London, Indiana University Press, pp. 357-388.

—(1989): I due corpi del Re. L'idea di regalità nella teologia politica medievale, Torino, Einaudi.

- (1990): I mosaici di Santa Maria dell'Ammiraglio a Palermo, Palermo, Istituto Siciliano di Studi Bizantini e Neoellenici.

- (2000): «La cattedrale di Cefalù. La cattedrale di Palermo e il Museo Diocesano. Mosaici profani», en Kitzinger, E. (ed.), I mosaici del periodo normanno in Sicilia, VI, Palermo, Istituto Siciliano di Studi Bizantini e Neoellenici.

LA DuCA, R. (2002): «Una cattedrale per Federico. Vicende Storiche del sarcofago di Federico II", Il sarcofago dell' imperatore. Studi, ricerche e indagini sulla tomba di Federico II nella Cattedrale di Palermo 1994-I999, Palermo, Regione Siciliana, pp. 303-315.

Lipinsky, Angelo (1973): «Le insegne regali dei sovrani di Sicilia e la scuola orafa palermitana", en Atti del Congresso Internazionale di Studi sulla Sicilia Normanna, Palermo, 4-8 dicembre 1972, Caltanissetta-Roma, Palermo, Sciascia, pp. 162-194.

Molina Lopèz, Laura (20I4): «La valorizzazione e diffusione del modello delle Tombe Regali di Palermo nella Penisola Iberica», en De Maria Manuel, S. y López De Corselas, P. (edd.), L'Impero e le Hispaniae da Traiano a Carlo V. Classicismo e potere nell'arte spagnola, Bologna, Bolonia University Press, pp. 56-64.

Pertusi, Agostino (1964): «Bisanzio e l'irradiazione della sua civiltà in Occidente nell'Alto Medioevo", en CISAM, XI Settimana di Studio, Centri e vie di irradiazione della civiltà nell'Alto Medioevo, Spoleto, Centro Italiano di Studi sull'Alto Medioevo, pp. 75-134.

Reilly, Lisa (2020): The Invention of Norman Visual Culture: Art, Politics, and Dynastic Ambition, Cambridge, Cambridge University Press.

Rossi VAIro, Giulia (20I8): «L'immagine dell'Altro nella scultura medievale funeraria europea: la tomba di Ruggero II di Sicilia e il monumento funebre di Dinis di Portogallo», en Albuquerque Carreiras, J. et. all. (edd.), Através do Olhar do Outro: Reflexóes acerca da sociedade medieval europeia (sécs. XII-XV), Tomar, Instituto Politécnico, pp. 157-202.

Salmaso, Linda (2019): «Il duomo di Monreale e le icone bizantine a mosaico", en Cantone, V. et al. (edd.), I mosaici della Sicilia normanna nella didattica universitaria. Dalla peer review alla costruzione delle competenze trasversali, Padova, Cleup, pp. 24I-24.

SChwinger, Anna Theresia (2006): «Sulla storia della trasmissione del parato d'incoronazione dei re ed imperatori del Sacro Romano Impero", en
Andaloro, Maria (ed.), Nobiles Officinae: perle, filigrane e trame di seta dal Palazzo Reale di Palermo (Vol. I-2), Catania, Maimone, pp. 244-255.

Scirea, Fabio (2020): «Designing a Visual Language in Norman Sicily: The Creation Sequence in the Mosaics of Palermo and Monreale», en Winkler, E.A et all. (edd.), Designing Norman Sicily. Material Culture and Society, Woodbridge, The Boydell Press, pp. 184-206.

VAGnONI, Mirko, (20II): «Problemi di legittimazione regia: “imitatio Byzantii”", en D’Angelo, E. y Leonardi, C. (edd.), Il papato e i Normanni: temporale e spirituale in età normanna, Atti del convegno di studi, Ariano Irpino 6-7 dicembre 2007, Firenze, SISMEL edizioni del Galluzzo, pp. 50-65.

- (2012): Le rappresentazioni del potere. La sacralità dei normanni di Sicilia un mito?, Bari, Edizioni Caratteri Mobili.

- (20I4): «I re normanni di Sicilia e i loro diplomi. Elementi di sacralità regia», en D’Angelo, E. y Ziolkowski, J. (edd.), Auctor et auctoritas in Latinis Medii Aevi litteris, Firenze, SISMEL, 20I4, pp. II65-II74.

- (2017): Dei gratia rex Sicilie. Scene d'incoronazione divina nell' iconografia regia normanna, Napoli, Federico II University Press.

- (2019): Epifanie del corpo in immagine dei re di Sicilia (II30-I266), Palermo, Palermo University Press.

Valenziano, Crispino y Valenziano, Maria (1979): La basilica cattedrale di Cefalu nel periodo normanno, Palermo, O Theologos.

Vergara Caffarelli, F. (2002): «Fonti documentarie per la storia delle tombe reali», Il sarcofago dell' imperatore. Studi, ricerche e indagini sulla tomba di Federico II nella Cattedrale di Palermo 1994-Ig99, Palermo, Regione Siciliana, pp. 316-340.

Travaini, Lucia (1973): La monetazione nell'Italia normanna, Roma, Ughelli. Tramontana, Salvatore (1993): Vestirsi e travestirsi in Sicilia. Abbigliamento, feste e spettacoli nel Medioevo, Palermo, Sellerio.

Duranti, Tommaso (2019): Obbedire a Danco. Scritti normanno-svevi, offerti in occasione del $70^{\circ}$ di Trombetti Budresi Anna, Ariano Irpino, Centro Europeo di Studi Normanni.

Tronzo, Wiliam (2006): «Il manto di Ruggero II», en Andaloro, Mario (ed.), Nobiles Officinae: perle, filigrane e trame di seta dal Palazzo Reale di Palermo (Vol. I-2), Catania, Maimone, pp. 443-446.

- (20II): «Il porfido nel Medioevo: racconti subliminali di un materiale», Seminario, Università Roma Tre, Roma.

- (2020): «The interplay of media: textiles, sculpture, mosaic,» Designing Norman Sicily», en Winkler E. et all. (edd.), Visual Culture and Society, Woodbridge, The Boydell Press, pp. 47-59.

Zug Tucci, Heleonore (2002): «Le incoronazioni imperiali nel Medioevo», en Cardini, Franco et. al. (edd.), Per me reges regnant. La regalità sacra nell'Europa medievale, Rimini-Siena, il Cerchio-Cantagalli, pp. II9-I36. 
